This item was submitted to Loughborough's Research Repository by the author.

Items in Figshare are protected by copyright, with all rights reserved, unless otherwise indicated.

Correcting for sub-grid filtering effects in particle image velocimetry data

PLEASE CITE THE PUBLISHED VERSION

PUBLISHER

(c) IOP Publishing Ltd

VERSION

AM (Accepted Manuscript)

LICENCE

CC BY-NC-ND 4.0

REPOSITORY RECORD

Spencer, Adrian, and David Hollis. 2010. "Correcting for Sub-grid Filtering Effects in Particle Image Velocimetry Data”. figshare. https://hdl.handle.net/2134/6089. 
This item was submitted to Loughborough's Institutional Repository (https://dspace.lboro.ac.uk/) by the author and is made available under the following Creative Commons Licence conditions.

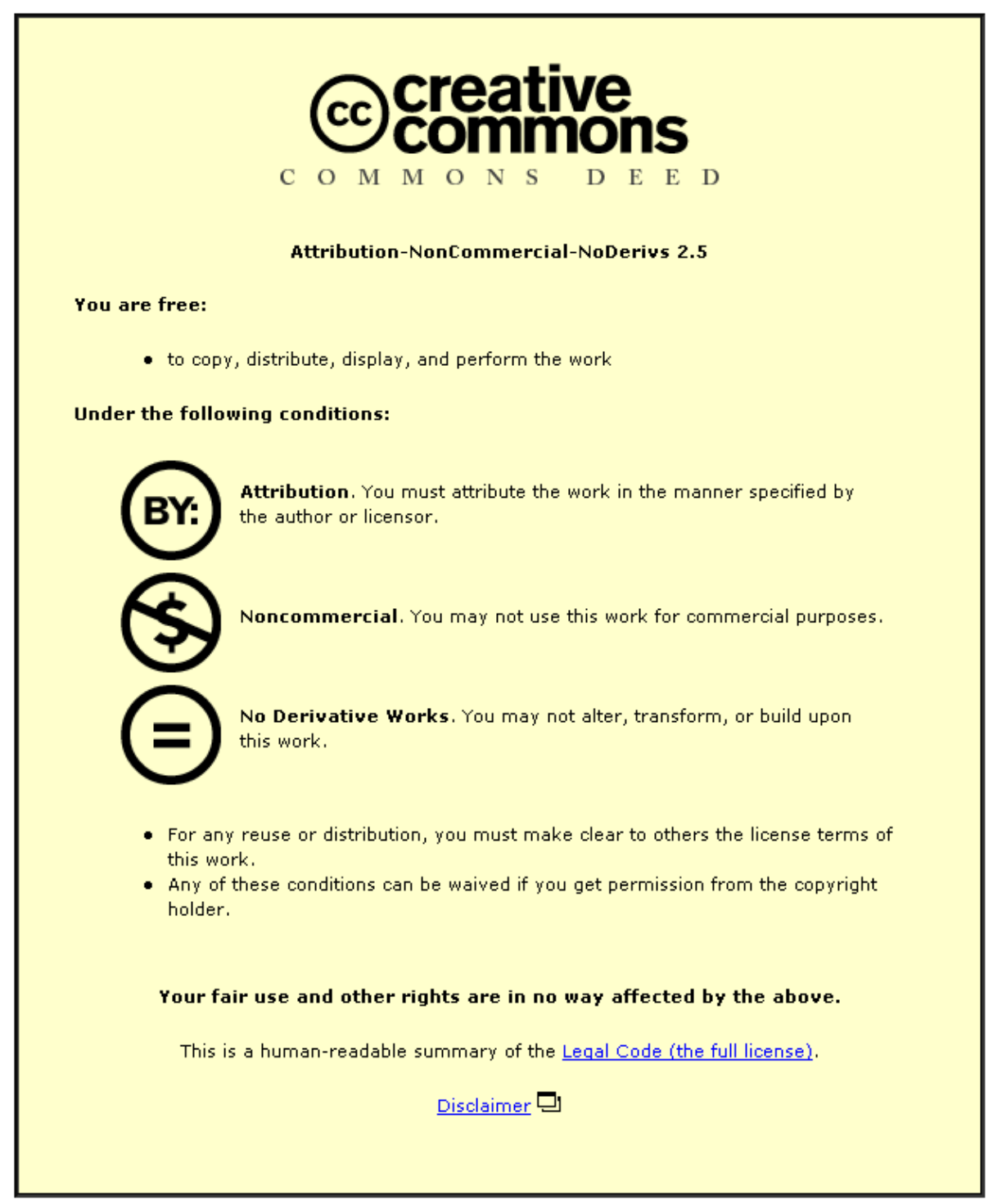

For the full text of this licence, please go to: http://creativecommons.org/licenses/by-nc-nd/2.5/ 


\title{
Correcting for Sub-grid Filtering Effects in Particle Image Velocimetry Data
}

\author{
Adrian Spencer and David Hollis ${ }^{1}$ \\ Department of Aeronautical and Automotive Engineering, \\ Loughborough University, Leicestershire, LE11 3TU
}

\begin{abstract}
Particle Image Velocimetry methodology results in a spatial averaging of the real velocity field into a set of discrete measured velocities: one for each interrogation cell. In the absence of measurement noise this filtering process results in a reduction of the measured turbulent kinetic energy and other second order statistics of the velocity field. The reduction in this energy will naturally be dependent upon the amount of turbulent energy at lengthscales smaller than can be resolved by the interrogation cells that make up the measurement grid. This paper investigates the effects of sub-grid scale filtering on the second order statistics of velocity. Several experiments are reported for which interrogation cell size to turbulent integral length scale ratios were varied. In addition, synthetic turbulent velocity fields with known spatial correlation functions are used to support experimental results and provide calibration for the estimation of the level of sub-grid filtering. It is suggested that to accurately capture all turbulent kinetic energy using PIV the interrogation cell should be at least of order 10 times smaller than the integral lengthscale of the flow. A method is then provided to estimate the level of sub-grid filtering should the interrogation cell be larger than
\end{abstract}

\footnotetext{
${ }^{1}$ Now at LaVision UK, PO Box 1235, Faringdon, Oxon. SN7 7YH
} 
this limit up to around the size of the integral lengthscale. With interrogation cells larger than this lengthscale then sub-grid filtering is such that second order statistics are reduced by over $50 \%$ and it should be considered unwise to rely on any second order statistics from such a scenario, corrected or otherwise.

Keywords: PIV, Particle Image Velocimetry, Sub-Grid, Turbulence, Spatial Filtering, Integral Lengthscale, Reynolds Stress, Turbulent Kinetic Energy.

\section{Introduction}

Particle Image Velocimetry (PIV) is now a widely utilised measurement technique in the investigation of turbulent engineering flows. Its proven ability to capture time series of instantaneous, two- or three-component velocity data over a planar field of interest allows the fluid dynamist to analyse structures forming, passing through, and evolving through the measurement domain. PIV allows us to uncover fluid dynamic phenomena previously extremely difficult to detect with point measurement techniques, whilst also providing a very efficient means of measuring single- and multi-point statistical data such as time average velocities, Reynolds stresses and spatial correlation functions.

Often there is significant turbulent energy in the flow field being measured that is contained in lengthscales similar to, or smaller than, the interrogation cells into which the field of interest has been divided for PIV analysis. Because of this, reported second order statistics such as Reynolds stresses and turbulent kinetic energy will be contaminated by a spatial filtering effect. The purpose of this paper is to provide a method of estimating the magnitude of this sub-grid filtering effect. 
Although stereoscopic and even holographic PIV measurements are now possible, all of the data given (and hence setups) in this paper refer to planar, two velocity component, digital PIV. However, the findings and methodologies described should be easily extended to the higher-dimensional techniques.

\subsection{Particle Image Velocimetry}

In the PIV technique a laser light sheet illuminates a plane of interest within the flow field, which is seeded with suitable tracer particles. Usually a dual head pulsed laser is utilised allowing two laser pulses to be emitted separated by a short user-defined time, $\Delta \mathrm{t}$ (of order $0.1 \mathrm{~ms}$ ). Virtually all modern PIV systems utilise digital cameras to image the illuminated plane of interest and then cross-correlation algorithms are used to determine the particle pattern shift in time $\Delta \mathrm{t}$. For each measurement, the images $\mathrm{A}$ and B (from each laser pulse pair) are separately stored on the camera sensor. The series of double frame images are continuously downloaded to a PC where they are then stored for analysis.

Each image frame is discretised into a regular grid of square interrogation cells, which are typically $32 \times 32$ pixels. Therefore, for calculation purposes, the image consists of a grid of smaller interrogation cells. (For simplicity, at this stage no consideration of advanced methods such as adaptive grids, deformed grids, or interrogation cell overlap has been given, as they do not influence this discussion.) To ensure suitable signal to noise ratios (SNR), each interrogation cell should contain at least 5 particle images. The ideal number of particles has been debated for several years, and tends to have asymptoted towards a value of 5; a figure given by Raffel et. al. (1998) for cross-correlation PIV. It is true that the SNR may increase if more particles are present (within reason), and it is likely that in highly turbulent engineering flows the 
number of particles present will vary somewhat across the region of interest. It is for this reason that most PIV experimentalists will typically aim for 8 particles per cell, to avoid regions of particle sparseness resulting in poor SNRs. The cross-correlation of PIV images results in a series of vector fields described by $U_{i}(\mathbf{x}, t)$ which has a time average $\bar{U}_{i}(\mathbf{x})$, where $\mathbf{x}$ is a discrete 2-Dimensional field.

\subsection{Sub Grid Filtering}

A single velocity vector is calculated for each interrogation cell, determined by locating the centroid of the tallest correlation peak in the cross-correlation plane of images A and B for that cell. Therefore the velocity estimation is a volume average of the velocity in the interrogation cell (Adrian, 1991). If the flow within each cell were completely homogeneous, the correlation peak representing the particle group movement in that cell would be a single uniform spike that would give the true fluid displacement within a given sub-pixel accuracy. Unfortunately, completely homogeneous flows seldom exist and are of little interest in real engineering problems, and so one must consider the fluid motion within individual cells.

Volume averaging can cause a smearing of the velocity gradients in velocity fields with non-zero spatial second derivatives as described by Raffel et. al. (1998). However, this is purely due to a spatial resolution (or discretisation) of velocity distributions. Here we are concerned with the effect on higher order statistics, where instantaneous velocity gradients due to turbulence can be much higher and are associated with much smaller characteristic lengthscales such that the velocity curvature is certainly non-zero. Figure 1 begins to illustrate why this averaging causes Sub-Grid Filtering (SGF) to affect PIV results. The finite size of the interrogation cell effectively performs a low pass filter on the measured displacement of the particle 
group contained within it. Any fluctuation occurring on a scale smaller than the cell dimensions is filtered out, with the average displacement of the particle group being recorded (whether the mean, mode, median or an arbitrary weighted average represents the displacement depends on the relative motions within the cell). On an instantaneous basis, the resulting vector field shows only the structures resolved by the spatial resolution of the interrogation cells, and any second order time average statistics (rms, turbulent kinetic energy, etc.) calculated will be filtered versions of the true values; a fact often overlooked or misinterpreted.

This type of 'group' averaging does not occur in LDA measurements, despite the LDA measurement volume often being of a similar size to the PIV interrogation cell. This is because LDA records one velocity measurement for each single particle passing through the measurement volume producing a valid Doppler burst, despite the fact that there may be more than one particle within the measurement volume at any one instant. The PIV methodology on the other hand relies on group averaging to allow accurate (sub-pixel) particle displacements to be calculated. The effect of 'velocity gradient broadening' (Durst et al, 1976) in LDA refers to the affect on statistics due to the velocity data being calculated from particles passing through any part of the measurement volume, across which there may be a velocity gradient in the time average. Again this is a spatial resolution issue which is being delineated from the effects of sub grid filtering on second order statistics discussed here. Indeed, in PIV this issue can be addressed by using a 'deformed grid' methodology that attempts to remove the effects of velocity gradients from the analysis (see Fincham and Delerce 2000). This method is adopted in this work as the authors believe this maximises the accuracy of the resultant velocity measurement. 
For a fixed camera sensor size and interrogation cell dimension in pixels, the object Field of View (FoV) determines the real physical dimension of each interrogation cell in the object plane. Therefore, a simple answer to the SGF problem is to perform PIV measurements using FoVs small enough to accurately capture the smallest motions. Although this may be feasible in some cases, often the fluid dynamicist is interested in both large instantaneous motions and accurate time average statistics. Repeating measurements at varying FoVs is an option, although this is time consuming and often prohibitive. It should also be appreciated that to resolve all turbulent motions the cell dimension should approach the Kolmogorov scale, $\eta$. From a modeling perspective Yeung and Pope (1989) state that, for Direct Numerical Simulations (DNS), the criteria $\Delta X \leq 2.1 \eta$ is necessary to fully resolve motions on all scales, where $\Delta X$ is the cell dimension. With particle sizes often exceeding the Kolmogorov scale (especially for water flows) this criteria is clearly unrealistic for conventional PIV. Fortunately the proportion of turbulent kinetic energy at these very small scales is usually very low. Another alternative is dual resolution PIV (Adrian, 2003). Here, simultaneous measurements are made using two (or more) cameras imaging different FoVs with one covering the larger 'resolved scale field' and the second imaging the 'sub-gridscale field'. However the experimental setup becomes more expensive and complicated with issues of optical access and image calibration or distortion being required.

\subsection{Previous Work}

In most PIV experiments all turbulent scales will not be resolved, and if supplementary small FoV PIV or point measurement experiments are unfeasible to attain the accurate statistical data, a means of dealing with the problem is essential. 
Those who recognise the effects of SGF generally approach the challenge in one of three ways:

i) Claim that all scales have been resolved via the use of sufficiently small interrogation cells.

ii) Attempt to recover the sub-grid scales by enhanced sub grid particle tracking algorithms.

iii) Attempt to estimate the loss of sub-grid fluctuations.

Comparisons between rms data from PIV and LDA have been given in the available literature (e.g. Baldi et. al., 2002; Finzenhagen et. al., 1999) to attempt to prove the accuracy of the PIV setup. However, the PIV data shown in these experiments where the interrogation cell sizes are typically of same magnitude as the integral length scales - is up to $30 \%$ lower than the equivalent LDA values in some areas of the flow. In other regions the data from the techniques are in close agreement, possibly due to the difficult flow scenario and insufficient local seeding resulting in poor SNR and the associated errors causing elevated rms velocities: The 'right' answer being obtained for the wrong reasons (high measurement noise).

Some authors do report PIV data collected at sufficient FoVs to resolve (almost) all turbulent scales. Lindken et. al. (2002) use a microscope PIV setup to enable cell sizes of as small as $42 \mu \mathrm{m}$. In their investigation, the Kolmogorov scale was around $5 \mu \mathrm{m}$, and hence they would clearly record almost all of the turbulent motions. However, with such a challenging configuration they reported a maximum of only $80 \%$ 'good' vectors. It is suspected that it is for this reasons that the PIV rms 
velocities are slightly higher than the equivalent DNS data (the relative error may increase the measurement noise and hence reported rms velocity).

A large proportion of workers in the field have concentrated on recovering the sub grid fluctuations via so called 'super-resolution' PIV - a natural pursuance to improve the technique given PIV's youth relative to the classical measurement techniques. Super-resolution PIV essentially attempts to recover the sub-grid fluctuations by particle tracking within cells (Wernet, 1999; Wernet, 2001; Stitou and Riethmuller, 2001) or adaptive multipass gridding down to particle diameter sizes (Hart, 2000). The concept of super-resolution PIV was introduced by Keane et. al. (1995), and Stitou and Riethmuller (2001) base their efforts on this initial work using conventional cross-correlation PIV followed by sub grid particle tracking within individual cells. They report promising velocity data although state that no quantitative estimation of the quality of the measurement can be defined. They conclude their work stating that extensive validation is required to answer the question "what are we measuring?" Wernet (1999) takes the concept a step further by introducing fuzzy logic principles to determine the correct particle pairing on the subgrid scale. It was found that "in practice, the particle pair operation has a success rate of approximately 30\%" which would have drastic consequences on the sub grid turbulence recorded in the event of false data being included in the statistics calculation. No rms data was presented in either of these sub-grid PTV documents. The idea of sub-grid particle tracking is logical, and the attempts have had some success but encounter a common obstacle: PIV requires at least 5 particles within each interrogation cell to have a high enough confidence in the recorded correlation peak certainly for displacements to sub-pixel accuracy. Given a constant seeding density, the effect of reducing the cell size simply reduces the number of particles present in 
each cell, in turn reducing the strength of the correlation peak and hence the confidence in that peak and displacement accuracy. The additional risk is that, at these sub-grid scales, no particles exist to track. Therefore all of the methods result in higher errors on the data because of the effects of noise, and elevated probability of false particle pairing, with the consequence of inflated rms values.

Sharp and Adrian (2001) calculate dissipation rates around a Rushton Turbine and use the Smagorinsky model; usually associated with sub-grid modelling in LES calculations, to estimate turbulent kinetic energy dissipation on the sub-grid scale. They find that in their experiment PIV captures at least $70 \%$ of the true dissipation. Saarenrinne et. al. (2001) use the Helland model spectrum to estimate the amount of turbulent kinetic energy (TKE) and dissipation rate recorded for a given cell size, finding that the spatial resolution should be around $20 \eta$ in order to record $95 \%$ of the TKE. In most flows of engineering interest this is a difficult criteria to meet. However this criteria is probably more applicable to low turbulence intensity flows with velocity spectra similar to model turbulence in late stages of decay. Here the interest is to take measurements where there is high turbulence intensity, significant regions of production and a large range in turbulent scales, with the large scales being highly anisotropic.

Hoest-Madsen and Nielsen (1995) [to be referred to as 'HMN' throughout] gave a theoretical examination of the problem of sub grid filtering and PIV, presenting a theoretical analysis by which the effect of filtering on the rms velocity could be related to the integral length scale and cell size. As with the work of Saarenrinne the analysis of HMN relies on model spectra based not on the Kolmogorov scale but on the Taylor and, more importantly, integral length scales. Initial studies (Hollis et. al., 
2001) comparing PIV and LDA data have suggested that the HMN correction could prove a useful basis of correcting PIV statistics for the effect of sub-grid filtering. However, the theory presented by HMN makes assumptions of the spatial correlation shape and also requires a priori knowledge of the flow length scale. In addition, no experimental validation of HMN theory was presented. The objective of this work was therefore to build on the HMN theoretical approach, and develop an automated methodology for the correction of PIV rms velocity data in a way that does not require additional measurement techniques nor require several PIV measurements at differing magnifications.

\section{Sub Grid Filtering Theory}

The theory of Hoest-Madsen and Nielsen (1995) is based on homogenous isotropic turbulence. Exactly isotropic turbulence does not exist in real flows (Hinze, 1959) but many flows have nearly isotropic local turbulence, and many theories are based on the assumption of isotropy. The notion that a significant number of non-trivial flow problems have near isotropic turbulence on a local (i.e. at sub-grid) scale is exactly what needs to be considered in the problem faced. The assumption of homogeneity and isotropy need only be true inside individual cells because the 'local' effect of filtering on individual vectors is what is being considered.

It is stated in Hoest-Madsen and Nielsen (1995) that they 'consider gradients due to turbulent motion', and they do so by looking at the effect turbulent statistics, rather than SNRs of PIV data. The latter has actually been considered by Scanaro and Reithmuller, (2000) who find that higher SNRs are achieved by utilising adaptive gridding to deform the image according to the velocity gradients across cells (calculated on the preceding cross-correlation pass). The higher SNR is achieved 
because broadening of the correlation peak due to velocity gradients is avoided. This technique also increases the accuracy of the mean velocities calculated, especially in highly sheared flows (and is utilised in the processing of all experimental data presented in this paper). However, this deformation does not increase the fundamental spatial resolution because the overall effective interrogation cell size remains approximately the same as a non-deformed scheme. Hence the velocity recorded at the interrogation cell centre is not the true velocity at that location but a spatial average over approximately one interrogation cell. The approach of the HMN technique actually attempts to account for the small-scale motions occurring on a subgrid scale, rather than merely 'considering gradients.'

Hoest-Madsen and Nielsen (1995) quote formulae from Hinze (1959) which provide a statistical model of homogeneous, isotropic turbulence, on which they base their analysis. The analysis hinges on correlation functions $\left(\mathrm{R}_{\mathrm{ij}}\right)$ relevant to the early and final stages of turbulence decay. These are given in terms of the integral length scale $\left(\mathrm{L}_{\mathrm{ij}}\right)$, and Taylor micro scale $\left(\lambda_{\mathrm{ij}}\right)$ of the flow with respect to the interrogation cell size $\Delta \mathrm{x}:$

$$
\mathrm{R}_{\mathrm{ij}}=\mathrm{e}^{\left(-\Delta \mathrm{x}_{\mathrm{k}} / \mathrm{k}_{\mathrm{ij}}\right)} \quad[1] \quad \mathrm{R}_{\mathrm{ij}}=\mathrm{e}^{\left(-\Delta \mathrm{x}_{\mathrm{k}}^{2} /{ }^{\mathrm{k}} \lambda_{\mathrm{ij}}^{2}\right)}
$$

Each of these equations is then used along with formulae representing the volume weighting and random errors in PIV measurements to numerically calculate the two curves, shown in Figure 2, describing the ratio of measured to true rms velocity. Thus, assuming the flow has a particular correlation function and that the flow lengthscale is known, it is possible to determine the extent of sub-grid filtering. However, determining the lengthscale is not trivial since SGF will also contaminate its 
evaluation from the PIV data. Therefore this theory is not complete for correcting PIV data without independent measurement of the lengthscale.

\section{Experimental Method}

All but one of the experiments reported in this paper were carried out in a vertically flowing water analogy facility. The first three configurations described here are used to demonstrate and validate the sub-grid filtering theory described above. The facility comprises two concentric cylindrical tubes, thus creating a 'core' $\left(\mathrm{r}_{\max }=45.0 \mathrm{~mm}\right)$ and an 'annular' flow $\left(r_{\min }=50.0 \mathrm{~mm}, r_{\max }=70.0 \mathrm{~mm}\right)$. The inner tube of the working test section can be interchanged. A blank section can be used to completely separate the flows (as in cases 1 and 2 below), or it can be replaced with a section containing porosity to simulate a generic gas turbine combustor flow field for example (as in case

3). The test section is fed from a constant-head tank to maintain a very stable flow rate through the rig. Further details on the test facility can be found in Spencer and McGuirk (2001). The test cases are illustrated in Figure 3; notice that the orientation has been rotated by $90^{\circ}$ for convenience.

\subsection{Case 1: Turbulent Pipe Flow}

Two points were chosen in the core flow; point $\mathrm{A}$ at the centreline, and point $\mathrm{B}$ close to the boundary layer, where the mean velocity was $72 \%$ of the centreline value. These points are approximately 18 pipe diameters downstream of the pipe inlet which consisted of a $30^{\circ}$ conical contraction. 


\subsection{Case 2: Flow Over a Double Faced Step}

A square section cylindrical hoop $(7 \mathrm{~mm} \times 7 \mathrm{~mm})$ was attached to the inner wall of the annulus, effectively creating an axisymmetric channel without the complication of sidewall effects. With a large inner tube radius to step height ratio this setup is close to planar two-dimensional. Three points of interest were chosen; the centre of the recirculation downstream of the step, a point in the free shear layer, and one in the relatively undisturbed free-stream. Figure 4 illustrates the time average flow field and points of interest.

\subsection{Case 3: Cylinder Wake Flow}

In this case the core pipe section incorporated 6 equi-spaced admission holes of $20 \mathrm{~mm}$ diameter, located $60 \mathrm{~mm}$ downstream of 6 equi-spaced cylindrical obstructions positioned in line with the ports. The annulus flow is accelerated around the cylindrical obstruction and causes an elongated reverse flow region due to the presence of the port admitting flow into the core. Two points of interest were studied for this setup as shown in Figure 5; one in the reversed flow of the wake, and the second in region experiencing the vortex street shedding.

The experimental setups used for the validation of the sub-grid filtering theory (cases 1-3 above) are summarised in table 1. Application of the sub-grid filtering theory is demonstrated on two further test configurations. The first of these is based on the jet impingement region created by the inflowing jets of test case 3, though the cylindrical blockages are removed to provide a circumferentially uniform annulus feed flow. Finally the method is applied to measurements on a fully featured sector rig of a gas turbine combustor. This final application is non-reacting but the isothermal airflow passes through the rig to match as closely as possible engine Reynolds number. 


\subsection{Instrumentation}

A LaVision PIV system is used to collect most data in this paper and comprises of a 50mJ New-Wave Solo Nd:YAG laser and Kodak E.S.1.0 camera operating at a data rate of $15 \mathrm{~Hz}$. Synchronisation of the system, and collection and processing of the data was done using the DaVis 6 software.

For all experimental setups two or three points of interest were chosen. Series of data at varying FoVs were then acquired to give a suitable variation in the $\Delta \mathrm{X} / \mathrm{L}$ ratio. The seeding density per cell was maintained constant regardless of FoV: i.e. the physical seeding density was increased as the FoV was decreased to maintain an average of 8 particles per $32 \times 32$ pixel cell. The seeding used was $20 \mu \mathrm{m}$ Polyamid which is approximately neutrally buoyant in water and is known to faithfully follow the flow. For the final airflow test presented an oil mist was used for seeding the airflow which produced droplets with a diameter of approximately $1 \mu \mathrm{m}$.

For validation purposes a 1-component LDA system was also employed. It comprised a He-Ne $20 \mathrm{~mW}$ laser and Dantec photomultiplier operated in forward scatter achieving data rates of $1.5 \mathrm{kHz}$. Delivery optics produced a control volume with major and minor axis of 3 and $0.3 \mathrm{~mm}$ with a fringe spacing of $3.28 \mu \mathrm{m}$. Typically a $0.4 \mathrm{MHz}$ frequency shift was used to remove directional ambiguity and the signal was analysed using a TSI IFA 550 processor. For further details of this system see Spencer and McGuirk (2001).

\subsection{Experimental Accuracy}

In all of the experiments described 4 blocks of 600 image pairs were used to determine the velocity statistics. With 2400 independent velocity samples at each 
point it is possible to determine the standard errors on the first and second moments. For typical turbulence intensities (of around $10 \%$ in the reported experiments) the errors in the mean and rms velocity are $1 \%$ and $4 \%$ respectively with a $99 \%$ confidence level. A check on the convergence of the PIV data at selected points showed that the $4 \%$ error on the rms velocity was conservative and agreement between repeat tests agreed to better than $3 \%$.

Bias errors on the velocity measurements are considered minimal, since all checks against other time average velocity data available (principally LDA) were in good agreement. Moreover, bias errors will not contribute to the second moment statistics that are discussed in this paper and are therefore not considered further.

Random errors, or measurement noise, will artificially contribute to the second moment statistics. The authors have minimised sources of noise as much as possible such that the effect of noise is not significant compared to the considerable turbulence levels present in the test cases presented. This has been done predominantly by ensuring initial image quality is high, following best practices, to obtain at least $98 \%$ first choice vectors. Advanced processing schemes were also used for all experiments and utilised adaptive gridding with $64 \times 64$ pixel initial cell size reducing to $32 \times 32$ pixel final cell size, where two iterations were performed as part of the adaptive scheme to achieve maximum displacement accuracy. Deformed grids were also employed to maximise signal to noise ratios and minimise the effect of curvature of the velocity gradients across individual cells. Finally, checks on the resulting data were carried out to ensure that peak locking and associated problems were not occurring. It is the authors opinion that the combined error due to statistical 
convergence and random error on reported rms velocities is therefore $5 \%$ within $99 \%$ confidence.

\section{Data Reduction}

\subsection{Measured Lengthscale}

The spatial correlation function is calculated using:

$$
R_{i j}(\mathbf{x}, \mathbf{r})=\frac{\overline{u_{i}(\mathbf{x}, t) u_{j}(\mathbf{x}+\mathbf{r}, t)}}{\sqrt{\overline{u_{i}(\mathbf{x}, t)^{2}}} \sqrt{\frac{u_{j}(\mathbf{x}+\mathbf{r}, t)^{2}}{2}}}
$$

Where subscripts $\mathrm{i}$ and $\mathrm{j}$ refer to any axis direction, $\mathrm{r}$ is the displacement vector, and $\mathrm{u}$ is the Reynolds decomposed velocity: $u_{i}(\mathbf{x}, t)=U_{i}(\mathbf{x}, t)-\bar{U}_{i}(\mathbf{x})$.

The measured integral lengthscale in direction $\mathrm{k}$ is then calculated from:

$$
{ }^{\mathrm{k}} \mathrm{L}_{\mathrm{ij}}\left(\mathbf{x}, \Delta \mathrm{x}_{\mathrm{k}}\right) \equiv \int_{0}^{\infty} \mathrm{R}_{\mathrm{ij}}\left(\mathbf{x}, \Delta \mathrm{x}_{\mathrm{k}}\right) \mathrm{d} \mathrm{x}_{\mathrm{k}}
$$

Where $\Delta \mathrm{x}_{\mathrm{k}}$ is the displacement of vector $\mathrm{r}$ along the $\mathrm{k}$ axis. The lengthscale can thus be considered a tensor, and the commonly referred to 'integral lengthscale' is the longitudinal value, where $i=j=k$, taken in the streamwise direction. Although the integral is mathematically described as being from the origin to an infinite displacement, it is commonly accepted practice to replace this with the first displacement crossing point.

Although the above formulae are well known, they have seldom been applied to experimental data due to the complexity in using them with point based measurements. PIV data is ideal for the application of the formulae, but is not a 
straightforward task: the calculation is also subject to errors associated with sub grid filtering. In addition, close to measurement plane edges that are flow inlets and outlets, part of the correlation curve can be curtailed prior to crossing the $\mathrm{x}_{\mathrm{i}}$-axis. Measures were therefore taken to avoid this influencing the calculated lengthscales by using a model correlation function (using a best fit of equation [1]) to estimate the missing portion of the curve where necessary. This illustrates a prime example where large fields of view are required to capture integral length scales, but in so doing the field of view is too large for the effects of sub grid filtering to be ignored.

\subsection{Actual Lengthscale}

To determine the actual integral lengthscale from the measured value (as calculated above) an understanding of the effect of sub-grid filtering on the correlation function and hence lengthscale had to be understood. This was done by examining synthetically generated velocity fields which had analytically defined spatial correlation functions which were then manually filtered by known amounts before applying the data reduction techniques as described. By comparing the specified correlation function to those calculated from the synthetic data, at various levels of filtering, a lengthscale correction could be determined. Moreover using the wellprescribed synthetic data it was also possible to independently check the results of HMN with respect to rms velocity correction in a similar fashion.

\subsubsection{Synthetic Data Generation}

The technique of Klein et. al. (2003) uses digital filtering to generate velocity fields and takes into account cross correlations between velocity components. The methodology of Klein et. al. was developed to generate inlet boundary conditions for 
time resolved (LES) CFD predictions. A velocity signal $U_{i}$ is generated that possesses prescribed statistics such that at any location (and given a large enough sample);

$$
\overline{U_{i}}(x)=0 \quad \overline{U_{i} U_{j}}(x)=\delta_{i j}
$$

These 'random number' velocity fields (with no spatial or cross-correlation) are then convolved with a filter to produce a spatially correlated field, $\hat{U}_{j}(x)$, with a prescribed correlation function shape, such as those given in equations 1 and 2. Here the convolution of the velocity and filter is actually done in Fourier space for efficiency which is possible because for any given velocity set the filter is invariant to location since the turbulence field is homogenous. The following transformation on the velocity field, $\hat{U}_{j}(x)$, is then performed:

$$
U_{i, \text { real }}(x)=\bar{u}_{i}(x)+a_{i j} \hat{U}_{j}(x)
$$

Where $a_{i j}$ is determined in order to give the desired Reynolds stresses. The integral lengthscale and statistical parameters are chosen, and the digital filtering technique produces the velocity fields, $U_{i, \text { real }}$, with prescribed mean velocity $\overline{u_{i}}(x)$, Reynolds Stresses $\left(\overline{\mathrm{u}_{\mathrm{i}} \mathrm{u}_{\mathrm{j}}}\right)$ and correlation functions $\left(\mathrm{R}_{\mathrm{ij}}\right)$. Using this method data with spatial correlation shapes of exponential and quadratic forms (as given in equations $1 \& 2$ ) has been generated. However, anisotropic lengthscales have only been investigated with the quadratic form - in the exponential data the prescribed lengthscale is invariant to direction. In all cases the velocity statistics and correlations have been chosen to be invariant to $\mathbf{x}$. 


\subsubsection{Synthetic Data Filtering}

Spatial averaging was carried out on the synthetic velocity data by applying a local $\mathrm{NxN}$ matrix averaging algorithm, thus creating several new sets of filtered velocity fields $\widetilde{U}_{\mathrm{i}}\left(\mathrm{x}_{1}, \mathrm{x}_{2}\right)$;

$$
\tilde{U}\left(x_{1}, x_{2}\right)=\frac{1}{N^{2}} \sum_{x_{1}-r}^{x_{1}+\gamma} \sum_{x_{1}-r}^{x_{2}+r} U_{i, r e a l}\left(x_{1}, x_{2}\right)
$$

where $\mathrm{N}=2 \mathrm{r}+1$. The local spatial averaging used $\mathrm{N}$ ranging from 1 (the raw data) to 17 ( $\mathrm{r}=0$ to 8$)$, and hence a large range of filtered cell size to prescribed lengthscale ratios could be considered.

The ensemble average mean and rms velocity, and integral lengthscale are then calculated based on the entire domain of each of the filtered data series. This is a valid approach given the synthetic flow is homogeneous. By doing so, high confidence is attained in the ensemble average quantities due to the effective large sample size.

The $\mathrm{NxN}$ spatial box filter acts to create a local average over a discrete vector field for each instantaneous vector in the field. Thus sub-grid filtering is simulated because each vector is then locally averaged in accordance with the filter dimensions. This is a simplified version of the way in which the cross-correlation calculation of raw PIV images is performed, because factors such as particle sparseness and non-uniform particle sizes are not taken into account. The analysis is therefore not specific to PIV but it does give valuable insight into the effect of spatial filtering as a process in its own right, without other noise being present in the data. It is also an effective controlled means of testing the HMN theory. 
Indeed, several sets of synthetic particle image pairs have also been created and analysed using the data from the synthetic velocity fields described. Here randomly located particle image pairs have been generated which have been displaced in turbulent velocity fields described by the synthetic velocity data. This method is not described further since the results of this analysis were in agreement with the analysis on the raw velocity data, indicating that the image cross-correlation step introduces negligible error when dealing with near perfect images.

\subsubsection{Synthetic Data Results}

With no filtering of the synthetic data it would be expected, via data reduction of it, to retrieve the correlation function and lengthscale specified in its generation. A comparison of the prescribed model correlation function compared with that calculated from 1024 unfiltered synthetically generated samples showed agreement within $1 \%$. This was also true near to the edges of the region of interest since the assumed shape of the curtailed correlation function exactly matched that prescribed in the synthetic velocity creation.

The use of synthetic data allowed the convergence of the spatial correlation with increasing sample size to be studied, and an example of this is given in Figure 6. Based on many data sets, an expected error in the correlation function (for any point on the curve) was found to be best described by:

$$
\varepsilon_{\mathrm{R}}= \pm \mathrm{Z}\left(\frac{1}{\sqrt{\mathrm{N}}}\right)
$$

This equation is of the same form as the standard error estimates for mean and rms data, where $\mathrm{N}$ is the sample size and $\mathrm{z}$ is the confidence band parameter. It is thus 
seen that with sufficient sample size and spatial resolution it is possible to extract accurate integral length scales by integrating the calculated correlation function. Convergence of the lengthscale with increasing sample size is seen in Figure 7 for point A of test case 2. This effectively illustrates the impact of the error in the correlation function, described above, on the lengthscale determined by the integration of it. The convergence observed for all other points examined followed a very similar pattern with the lengthscale calculated from small samples tending to be biased toward larger values than that expected. Once several hundred samples were used the scatter in the lengthscale was more symmetric and comparison of the integral lengthscales calculated for various tests using 1200 samples showed a scatter of less than $4 \%$ about the expected value.

Collated in Figure 8 is the ratio of prescribed rms velocity against those calculated from the various filtered synthetic velocity data series. This allows the results from the filtered synthetic data study to be compared directly to the theory of HMN. Agreement between the HMN (Integral) and Synthetic (Exponential) curves is especially good (both use Eq. 1 to describe the basis correlation function). The comparison between the Taylor and Quadratic curves (Eq. 2) are also in close agreement for $\Delta \mathrm{X} / \mathrm{L}<1$, but the synthetic data tends to follow the exponential curve for higher ratios.

In the synthetic data, the true integral lengthscale $\left(\mathrm{L}_{\text {true }}\right)$ is always known a priori since it has been prescribed in the generation of that data. However, in experimental measurements this will not be the case. Evaluating the spatial correlation requires the fluctuating velocity components that are low pass filtered, it is therefore logical that the integral lengthscale calculated from the measured velocity data is also affected by 
SGF. Thus, the correction of PIV data using only curves such as those in Figure 8 is not possible.

\subsubsection{Lengthscale correction for sub-grid filtering}

Figure 9 shows the effect of the filter size upon the spatial correlation function, whereby increasing the filter causes the correlation curve to be smoothed, with the crossing point being delayed resulting in a greater area under the curve. This in turn affects the ratio of true to measured lengthscale, i.e. increased sub-grid filtering will increase the measured lengthscale. By compiling the results of the prescribed to calculated lengthscale for all of the levels of filtering on the synthetic data a resulting trend with varying $\Delta \mathrm{X} / \mathrm{L}$ can be found and is shown in Figure 10. It is again seen that the basis correlation function has a significant effect on these trends. In order to use the information in Figure 10 some iteration is required since the true lengthscale is not initially known, but the iteration process is found to converge quickly.

\section{Results: Validation of the Sub-Grid Filtering Correction}

The synthetic data has shown good agreement with the correction method proposed by Hoest-Madsen and Nielsen (1995) and also provided additional data that shows the effect of SGF upon measured integral lengthscales. However both HMN corrections use idealised correlation functions absent of the complexities of real flows. Therefore three experimental investigations were performed to estimate the validity of these assumed correlations. The 3 test cases were chosen for their convenient configurations in the facility available whilst at the same time representing a wide range of fluid dynamic phenomena ranging from undisturbed free stream flows, through strong free shear layers, to regions of swirl and recirculation. 
In this section the true lengthscale has been established from the most accurate measurement source available: the smallest FoV PIV data. In most cases this has also been validated against available LDA data to within 5\%. However at several points, near to time averaged free-stagnation points, the use of Taylor's frozen turbulence hypothesis is questionable since it is only applicable when convection velocities are much higher than fluctuating velocities. Results between PIV and LDA were therefore not in good agreement close to these points and it was evident that the smallest FoV PIV data was the most complete and consistent source of 'true' lengthscale information. By relying on the analysis of the synthetic data (Figure 10) it is possible to deduce that sub-grid filtering at the smallest FoV would increase the true lengthscale by less than $1 \%$ at any of the chosen points of interest and will have a negligible effect on the graph.

For all of the points of interest in the three test cases, the ratio of the PIV measured rms velocity to the true rms value, $u_{\text {meas }}^{\prime} / u_{\text {true, }}$ is plotted against the non-dimensional interrogation cell size, $\Delta \mathrm{X} / \mathrm{L}_{\text {true }}$ in Figure 11. Here $\mathrm{u}_{\text {true }}$ is determined from 1D-LDA measurements at these points. Although there is some scatter in the data it does agree well with both the HMN theory and the analysis of the synthetic velocity fields. Since it is the integral lengthscale that is extracted from the experimental data only the theoretical curves based on the exponential correlation function are shown. However, looking back to Figure 2, it is possible to see that most of the scatter lies in the area between the two HMN curves derived from quite different correlation functions. For this reason the authors choose to adopt a best fit polynomial line to the experimental data rather than rely on either of the theoretical curves. For $\Delta \mathrm{X} / \mathrm{L}_{\text {true }}<1.0$ then this best fit can be described by; 


$$
\mathrm{u}_{\text {meas }} / \mathrm{u}_{\text {true }}^{\prime}=0.2381\left(\Delta \mathrm{X} / \mathrm{L}_{\text {true }}\right)^{3}-0.395\left(\Delta \mathrm{X} / \mathrm{L}_{\text {true }}\right)^{2}-0.1155\left(\Delta \mathrm{X} / \mathrm{L}_{\text {true }}\right)+1.000
$$

Figure 12 shows how the true to measured lengthscale ratio varies for the selected points of interest in the three test cases again at a selection of fields of view. Here only a comparison to the synthetic data analysis is possible since this is not an area investigated by Host-Madsen and Nielson (1995). Scatter in the experimental data is again observed but it follows a very similar trend observed from the synthetic data analysis - and again this scatter is to one side of the 'theoretical' line. Thus rather than rely on the results of an idealised study the authors again adopt an empirical best-fit relationship. For $\Delta \mathrm{X} / \mathrm{L}_{\text {true }}<1.0$ then this best fit can be described by;

$$
\mathrm{L}_{\text {meas }} / \mathrm{L}_{\text {true }}=\mathrm{e}^{-0.4001(\Delta \mathrm{X} / \mathrm{Ltrue})}
$$

Evidence from additional tests by the authors and others also agree with the data in Figures 11 and 12 within the limits of scatter. These are not included for brevity and also since there is less confidence on these data with respect to the estimated lengthscales. One scenario, strongly swirling flows with high through measurementplane velocities, has been found not too agree within these bounds of scatter (Midgley et al, 2004). It is thought that the compromised interframe times required in this PIV setup introduced considerable noise and thus artificially increased the rms velocities recorded. This effect was most pronounced at large fields of view and it was seen that the level of sub-grid filtering was apparently minimal across the whole range of $\Delta \mathrm{X} / \mathrm{L}$ - an example of the correct answer being obtained due to the canceling of two errors.

The level of scatter in the experimental data of Figures 11 and 12 is explained by considering Figure 13. The actual correlation function for any point in a turbulent flow field will vary and it is unlikely to match either of the model functions used so 
far throughout this analysis. Typically $\mathrm{R}_{\mathrm{ij}}$ is found to have a shape that falls somewhere between the two models presented and can often be unsymmetrical, with Figure 13 as just one example. The best-fit lines to the data in Figures 11 and 12 do perhaps imply a particular basis correlation function (or family of) but the reverse analysis has not been done to determine what form this takes.

Evidence is also available to the authors that the effect of SGF on shear stresses follows a very similar trend to those effects on normal stresses (Hollis 2004). However this data is limited and not fully validated since the 1 component LDA system is unable to provide the shear stresses directly.

\section{SGF Correction Methodology}

Having demonstrated the validity of a HMN-type correction and calibrated the curves with a significant amount of experimental data, a methodology has been developed in a way that is possible to implement in an automated manner.

Figure 14 describes this methodology schematically, and can be implemented as a post processing exercise on PIV velocity data. For any given measurement set standard PIV analysis can be used to produce the velocity vector fields, U. Data reduction can then be used to derive statistics of these velocities, such as the mean and rms velocities: but the latter may be subject to errors introduced by sub-grid filtering. Without providing any additional information other than the original velocity data set it is possible to estimate the level of sub-grid filtering. Firstly, spatial correlations of the fluctuating velocity components must be calculated and integration of these allows the integral lengthscales to be calculated for each point. However, these 'measured' lengthscales could also be incorrect due to sub-grid filtering, but the data in Figures 10 or 12 can now be used to estimate this error. Here the authors choose to use the 
empirical relationship (a best fit to the scattered experimental data) of Figure 12. This effectively assumes a 'typical' correlation shape rather than one of the model functions. This step requires 2 or 3 iterations to converge, initially taking the measured lengthscale as the unknown true value. With the spatially resolved 'true' lengthscales now determined it is then possible to determine the local correction factors for the rms velocities from the data in Figures 8 or 11. Again the authors favour the use of empirical evidence rather than relying on model correlation functions. To correct $\mathrm{u}_{1}$ ' then ${ }^{1} \mathrm{~L}_{11}$ is used and similarly ${ }^{2} \mathrm{~L}_{22}$ for $\mathrm{u}_{2}$ '. With the 'correction factors' now available a choice may be made on what best to do with them. If the correction is less than $5 \%$ it may be fair to ignore it since this correction or error maybe smaller than the experimental accuracy. Indeed, in this instance the effect of the correction may also amplify experimental noise resulting in too high a value of rms velocity. If the correction is greater than $50 \%$ then it is fair to assume that a smaller FoV should be used to obtain more accurate second order velocity statistics. Between these values of 5-50\% the correction may be applied locally to the data or quoted as an estimate of the (spatially resolved) bias error.

\section{Results: Application of the SGF Correction}

A methodology had been developed and best-fit correction curves calibrated for a wide range of experiments. As a final test, application of the correction method to flows of interest to the authors was carried out. These test cases are ideal in that they contain features of a variety of sizes that produce a highly turbulent flow with lengthscales that vary considerably through the domain. The first flow utilises the same water analogy facility as detailed earlier. The second is an isothermal airflow 
facility containing a $45^{\circ}$ sector of a fully featured gas turbine combustor, with a Reynolds number high enough to be representative of real engine conditions.

The data presented are restricted to rms velocities because of the difficulties in acquiring a reliable estimation of the true integral lengthscales with which to compare the PIV derived values. As with previous measurements the seeding particle density is altered between FoVs to ensure around 8 particles per interrogation cell are obtained.

\subsection{Generic Combustor Geometry (Water Flow)}

Figure 15 shows the time average flow field in streamtrace form with shaded contours indicating $\mathrm{U}_{\mathrm{x}}<0$. The mass flow splits were chosen to emulate the primary zone of a gas turbine combustor. $50 \%$ of the annular flow is admitted into the core via 6 equispaced ports. This results in six jets impinging and bifurcating at the centre of the core and a crossflow velocity is set to give a jet to crossflow velocity ratio of 5 . A portion of the jet flow is reversed upstream against the incoming core flow, creating the toroidal recirculation zone. The flow is highly turbulent and three-dimensional. A profile through $\mathrm{r}=12 \mathrm{~mm}$ was taken and is provided in Figure 16. It compares LDA data (Spencer, 1998) and PIV results for the axial rms velocity. The PIV data includes both uncorrected (measured) and corrected values from a large FoV, and uncorrected data from a small FoV. Measured data has been included for the small FoV because in this case the effect of SGF is less than 5\% across the domain. The improvement via use of the correction method is obvious, with the resulting rms profile being in much better agreement with the LDA and small FoV data. The slight difference between LDA and (corrected) PIV is thought to be a very slight difference in flow conditions, to which the jet impingement point is very sensitive. This is supported by the fact that the corrected large FoV closely matches the small FoV data. 


\subsection{Fully Featured Combustor Sector (Air Flow)}

Figure 17 shows a schematic view of the type of geometry the fully featured combustor comprises. It includes dump diffuser, fuel injector, and chuted primary and secondary ports. In this case comparison of LDA (Griffiths, 2000) and PIV data is made across the inner primary port at $r=-44 \mathrm{~mm}$, given in Figure 18 (where $r=0$ is the flame tube centreline). This type of configuration presents a major challenge because of the large range of turbulent lengthscales throughout the flow field combined with high turbulent intensities. PIV derived integral lengthscales vary in the measurement domain from less than $1 \mathrm{~mm}$ to $12 \mathrm{~mm}$. To capture the full height of the combustor (around 150mm) it is clear that varying levels of sub grid filtering will take place. In all except the region of the leading edge of the port, the correction methodology brings the large FoV data in to close agreement with the small FoV and LDA data available. This correction is done using only the large FoV data - i.e. the lengthscales required for the correction are derived from this data. No information is required about the combustor sizes or from the small FoV measurements (or LDA data) provided the large FoV images are properly calibrated. Whilst there are some discrepancies between the three measurement sets in this challenging flow it is clear the uncorrected large FoV data stands alone in under reporting the true turbulence intensities by up to one third.

\section{Conclusions}

When PIV data is captured at a magnification such that the flow integral lengthscale is less than around 10 times the size of the interrogation cells then second order statistics calculated for the velocity field will be in significant error. This error is caused by the spatial filtering of all turbulent energy contained in eddies of scales similar to, or 
smaller than, the interrogation cells into which the PIV images are divided. Quantification of this error has been presented in this paper via three methods;

i) An analytical approach assuming model correlation functions, as presented by Hoest-Madsen and Nielson, (1995),

ii) An experimental approach where varying fields of view or comparison with LDA data has been used to determine the extent of sub-grid filtering on turbulent flows, and finally,

iii) Synthetic velocity data has been generated with known spatial correlation functions, from which the statistics of filtered versions of this data have been compared to the statistics of the unfiltered data.

All of these approaches agree well with regard to the extent of sub-grid filtering when the ratio of the integral lengthscale to interrogation cell size ratio changes. However, some variation in the magnitude of this error is dependent on the shape of the actual correlation function. For this reason an empirically derived estimation of the correction factor is suggested for use when the integral lengthscale is between one and ten times the size of the interrogation cell size. If the lengthscale is larger than this range it is suggested that the effects of sub-grid filtering will be less than $5 \%$ on the rms velocity and any correction is unnecessary (and perhaps undesirable since experimental noise could be amplified). If the lengthscale is smaller than this range then the measured rms velocity will be around $50 \%$ or less of the true value. For this reason any second order statistics, corrected or not, should be considered unreliable.

The application of the suggested sub-grid filtering correction method has been demonstrated to good effect on two combustor-related flows. These cases both 
contain high levels of turbulence at a wide range of lengthscales. In all cases the large field of view PIV data corrected for sub-grid filtering agrees much better with LDA and small field of view PIV results not affected by spatial filtering.

\section{Acknowledgements}

The work presented here was carried out under projects funded by EPSRC and RollsRoyce plc for which the authors express their gratitude.

\section{References}

Adrian, R.J. (1991) - "Particle-Imaging Techniques for Experimental Fluid Mechanics", Annual Review of Fluid Mechanics, Vol. 23.

Adrian, R.J (2003) - "Innovations in Flow and Particle Diagnostics", TSI Inc Seminar, Harrogate, 28th Jan 2003.

Baldi, S., Hann, D. and Yianneskis, M. (2002) - "On the Measurement of Turbulent Energy Dissipation in Stirred Vessels with PIV Techniques", Application of Laser Technology to Fluid Mechanics, 11th International Symposium.

Durst, F., Melling, A. and Whitelaw. J.H. (1976) - "Principlesand Practice of Lase Doppler Anemometry”, Academic Press Inc.

Fincham A. and Delerce G. (2000) - “Advanced Optimisations of Correlation Imaging Velocimetry Algorithms” Experiments in Fluids, Vol.29, pp.51-60. 
Finzenhagen, F., O'Doherty, T.O., Bates, C., Kremer, H. and Wirtz, S. (1999) "Statistical Analysis of PIV Data and Determination of Small Turbulent Scales in Highly Turbulent Flows", 3rd International Workshop on PIV'99, Santa Barbara, pp.519-525.

Griffiths, J.P. (2000) - "Measurements of the Flow Field in a Modern Gas Turbine Combustor", PhD Thesis, Loughborough University, UK.

Hart, D.P. (2000) - "PIV error correction”, Exp in Fluids, Vol.29, pp13-22.

Hinze, J.O. (1959) - "Turbulence: An Introduction to Its Mechanism and Theory", McGraw-Hill Book Company, Inc.

Hoest-Madsen, A. and Nielsen, A.H. (1995) - "Accuracy of PIV Measurements in Turbulent Flows", ASME Laser Anemometry, FED-Vol. 229.

Hollis, D. (2004) - "Particle Image Velocimetry in Gas Turbine Combustor Flow Fields", PhD Thesis, Loughborough University, UK.

Hollis, D., Spencer, A. and Carrotte, J.F. (2001) - "Statistical Analysis and Comparison of LDA and PIV Measurements for the Flow Over a Wall Mounted Obstacle", 9th Int. Conf - Laser Anemometry Advances and Applications, Limerick.

Keane, R.D., Adrian, R.J. and Zhang, Y. (1995) - "Super Resolution Imaging Velocimetry”, Measurement Science and Technology Vol.6, pp.754-763.

Klein, M., Sadiki, A. and Janicka, J. (2003) - “A Digital Filter Based Generation Of Inflow Data For Spatially Developing Direct Numerical Or Large Eddy Simulations", J. Comp Physics, Vol.186, pp.652-665. 
Lindken, R., Silvestro, F. Di., Westerweel, J. and Nieuwstadt, F.T.M. (2002) -

"Turbulence Measurements with Micro-PIV in Large Scale Pipe Flow", 11th Symposium on the Application of Laser Techniques to Fluid Mechanics.

Midgley, K., Spencer, A., McGuirk, J.J. (2004) - "Unsteady Flow Structures in Radial Swirler Fed Fuel Injectors”, Proc. ASME Turbo Expo 2004, ASME GT2004-53608

Raffel, M., Willert, C. and Kompenhaus, J. (1998) - "Particle Image Velocimetry", Springer-Verlag.

Saarenrinne, P., Piirto, M. and Eloranta, H. (2001) - "Experiences of turbulence measurement with PIV”, Meas. Sci. Tech., Vol.12, pp.1904-1910.

Scanaro, F. and Reithmuller, M.L. (2000) - "Advances in Iterative Multigrid PIV Image Processing”, Exp in Fluids [suppl], pp.S51-60.

Sharp, K.V. and Adrian, R.J. (2001) - "PIV Study of Small-Scale Flow Structure Around a Ruston Turbine”, Fluid Mechanics and Transport Phenomena, Vol.47, No.4. Spencer, A. (1998) - "Gas Turbine Combustor Port Flows", PhD Thesis, Loughborough University, UK.

Spencer, A. and McGuirk, J.J. (2001) - "LDA Measurements of Feed Annulus Effects on Combustor Liner Port Flows", Journal of Fluids Engineering, Vol.123, Part 2, pp.219-227.

Stitou, A., and Riethmuller, M.L. (2001) - "Extension of PIV to super resolution using PTV”, Measurment Science and Tecnology, vol.12, pp.1398-1403. 
Wernet, M.P. (1999) - "Fuzzy Logic Enhanced DPIV Processing Software", NASA/TM-1999-209274.

Wernet, M.P (2001) - "New Insights into Particle Image Velocimetry Data using Fuzzy Logic Based Correlation/Particle Tracking Processing”, Experiments in Fluids 30, pp.434-447.

Yeung, P.K. and Pope, S.B. (1989) - "Lagrangian Statistics from Direct Numerical Simulations of Isotropic Turbulence”, J. Fluid Mechanics, Vol.207, pp.531-586.

\section{List of Figures}

Figure 1. Illustration of the low pass filtering of the interrogation cell.

Figure 2. Theoretical Hoest-Madsen and Nielsen (1995) correction curves.

Figure 3. Experimental test Case 2 (left) and 3 (right) with PIV measurement planes indicated.

Figure 4. Time average description of the flow over a step (case 2).

Figure 5. Time average description of the cylinder wake flow (case 3).

Figure 6. Correlation function variation with increasing sample size.

Figure 7 Integral lengthscale convergence with increasing sample size - Case 2 Point B.

Figure 8. Effect of spatial filtering on synthetic rms velocity compared with HMN theory. 
Figure 9. Effect of filter size upon spatial correlation function.

Figure 10. Integral lengthscale trend with increased spatial filtering - synthetic data.

Figure 11. Sub-grid filtering effect on rms velocity - experimental data.

Figure 12. Sub-grid filtering effect on integral lengthscale - experimental data.

Figure 13. Comparison of experimental spatial correlation function and model functions - Case 2; Point B.

Figure 14. Schematic of sub-grid filtering correction methodology.

Figure 15. Time average streamtraces in generic combustor geometry.

(Shaded contours indicate negative axial velocity.)

Figure 16. Comparison of corrected and uncorrected data at $\mathrm{r}=12 \mathrm{~mm}$.

Figure 17. Combustor section view with comparison plot position indicated by the dashed line.

Figure 18. Comparison of corrected and uncorrected data at inner primary port.

NB: Fig 14 full page width - all others single column width. 


\begin{tabular}{|c|c|c|c|c|c|c|}
\hline Point & $\begin{array}{l}\text { Magnification } \\
\text { Range }\end{array}$ & $\Delta X / L$ Range & $\mathbf{u}_{1}{ }^{\prime}$ true $/ \mathbf{U}_{\text {ref }}$ & $\mathbf{u}_{2}^{\prime}{ }_{\text {true }}^{\prime} / \mathbf{U}_{\text {ref }}$ & ${ }^{1} \mathrm{~L}_{11}[\mathrm{~mm}]$ & ${ }^{2} \mathrm{~L}_{22}[\mathrm{~mm}]$ \\
\hline \multicolumn{7}{|c|}{ Case 1 : Pipe Flow : $U_{\text {ref }}=0.503 R e=50468$ (based on pipe diameter) } \\
\hline$A$ & \multirow{2}{*}{$0.060 \leq \mathrm{M} \leq 0.563$} & $0.0817 \leq \Delta X / L \leq 0.7658$ & 0.0272 & & 6.23 & \\
\hline B & & $0.0863 \leq \Delta X / L \leq 0.8090$ & 0.0994 & & 5.90 & \\
\hline \multicolumn{7}{|c|}{ Case 2 : Flow Over Step : $U_{\text {ref }}=0.340 R e=7581$ (based on channel height) } \\
\hline$A$ & \multirow{3}{*}{$0.114 \leq \mathrm{M} \leq 0.622$} & $0.0418 \leq \Delta X / L \leq 0.8969$ & 0.2705 & 0.2235 & 5.50 & 2.80 \\
\hline B & & $0.0921 \leq \Delta X / L \leq 1.0045$ & 0.4043 & 0.2705 & 2.50 & 2.50 \\
\hline C & & $0.0742 \leq \Delta X / L \leq 0.9132$ & 0.0620 & 0.0588 & 5.50 & 2.75 \\
\hline \multicolumn{7}{|c|}{ Case 3 : Cylinder Wake : $U_{\text {ref }}=0.552 R e=12308$ (based on cylinder diameter) } \\
\hline A & \multirow{2}{*}{$0.065 \leq \mathrm{M} \leq 0.430$} & $0.1256 \leq \Delta X / L \leq 1.1328$ & 0.2861 & 0.2843 & 3.90 & 5.30 \\
\hline$B$ & & $0.0652 \leq \Delta X / L \leq 0.8033$ & 0.3278 & 0.2898 & 5.50 & 10.0 \\
\hline
\end{tabular}

Table 1. Experimental Setups. 


\section{Figures}

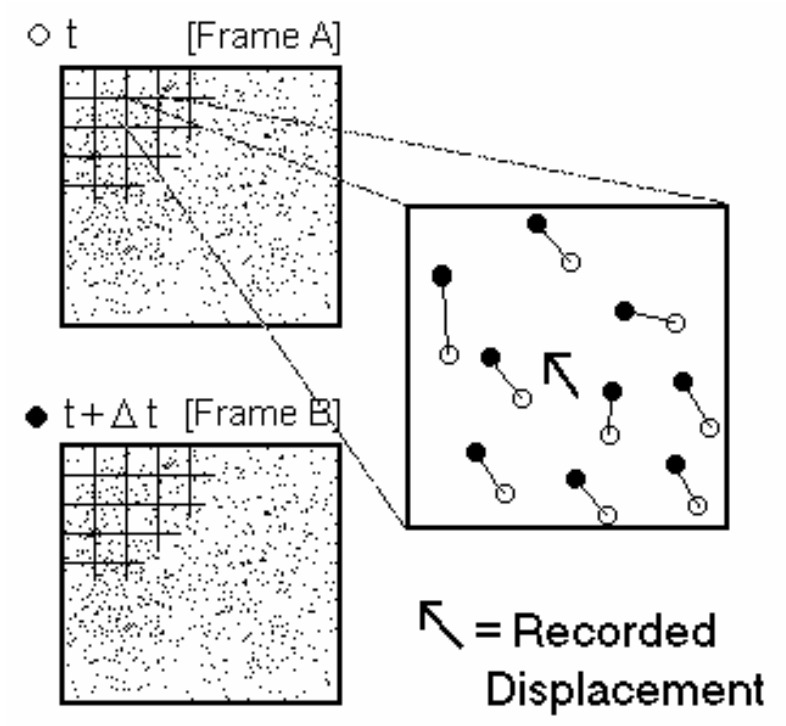

Figure 1. Illustration of the low pass filtering of the interrogation cell.

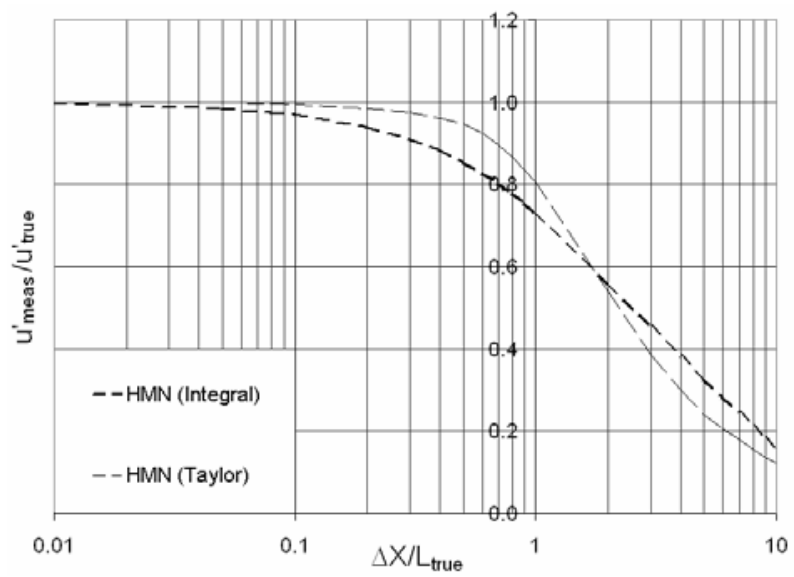

Figure 2. Theoretical Hoest-Madsen and Nielsen (1995) correction curves.

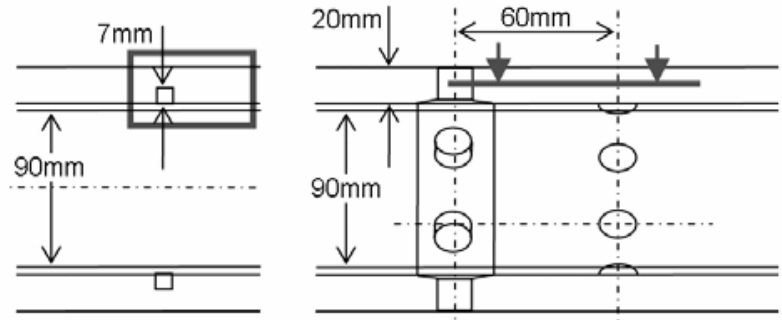

Figure 3. Experimental test Case 2 (left) and 3 (right) with PIV measurement planes indicated. 


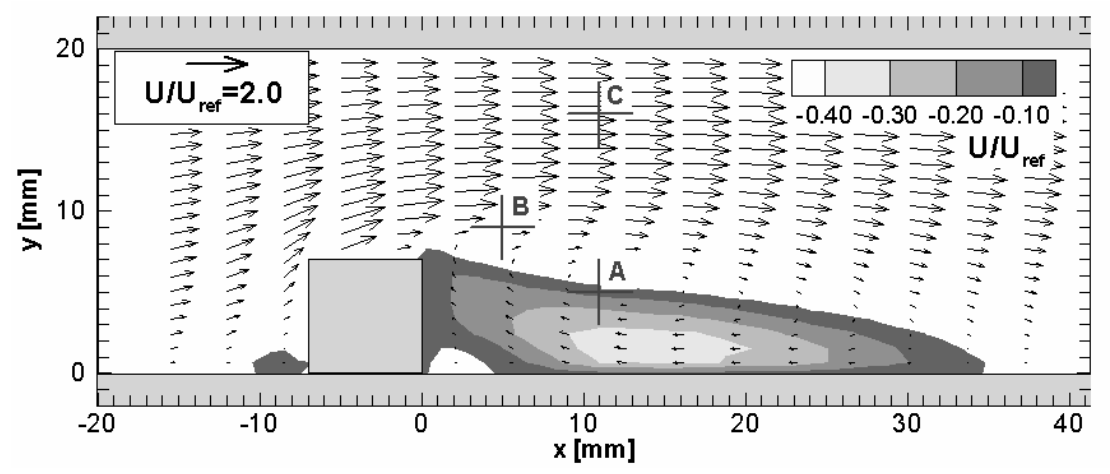

Figure 4. Time average description of the flow over a step (case 2).

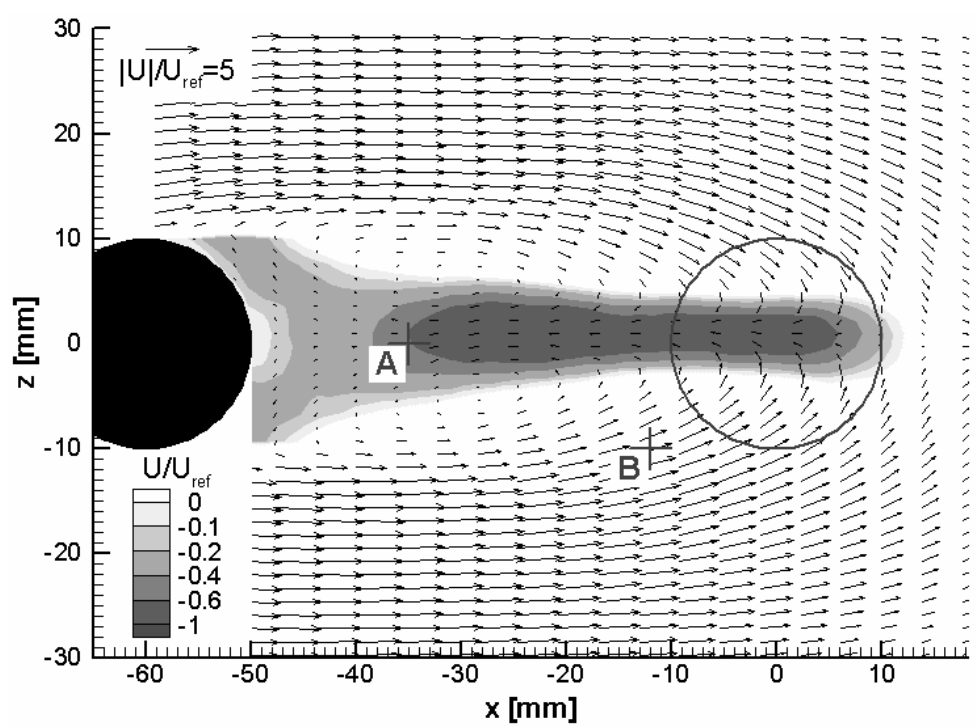

Figure 5. Time average description of the cylinder wake flow (case 3).

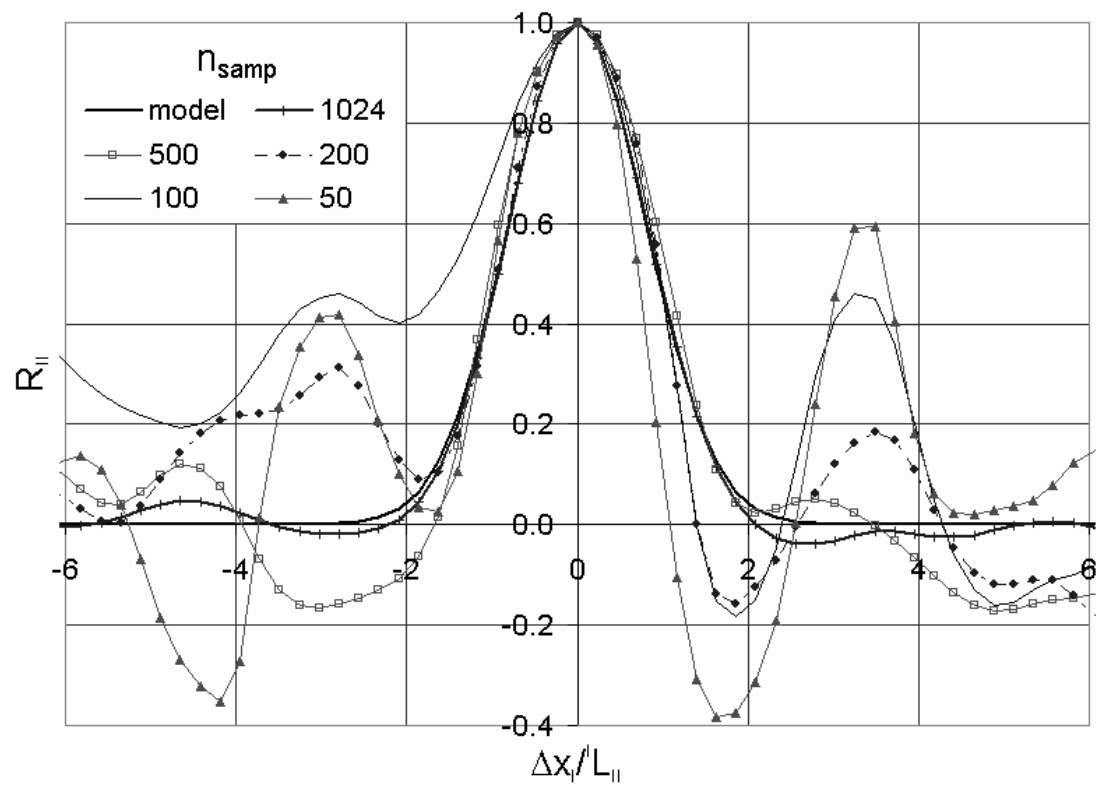

Figure 6. Correlation function variation with increasing sample size. 


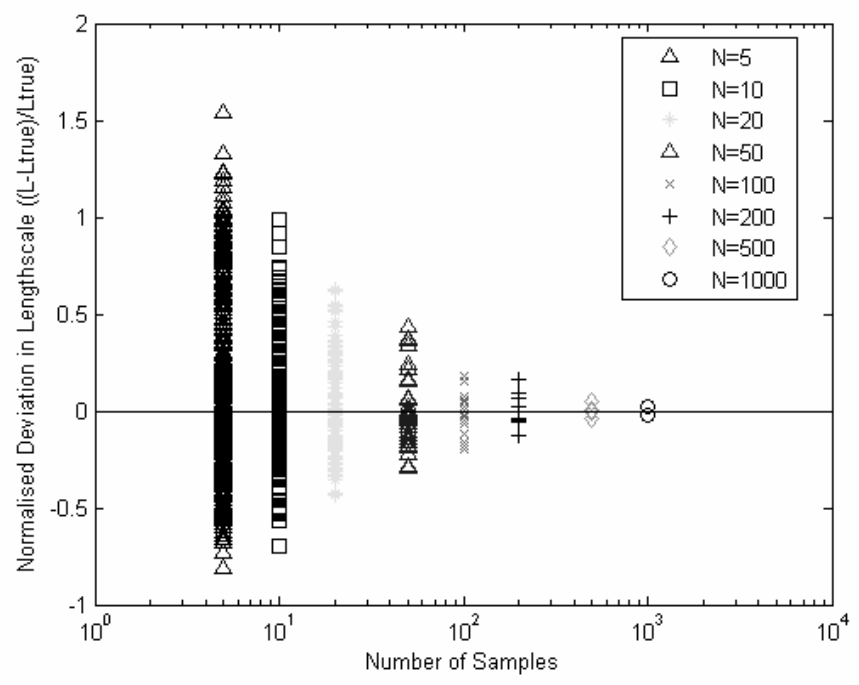

Figure 7 Integral lengthscale convergence with increasing sample size Case 2 Point B.

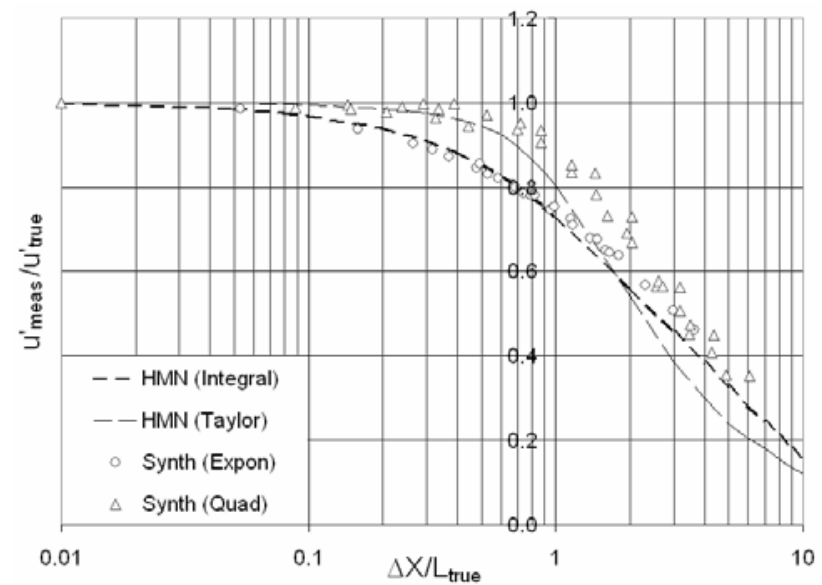

Figure 8. Effect of spatial filtering on synthetic rms velocity compared with HMN theory.

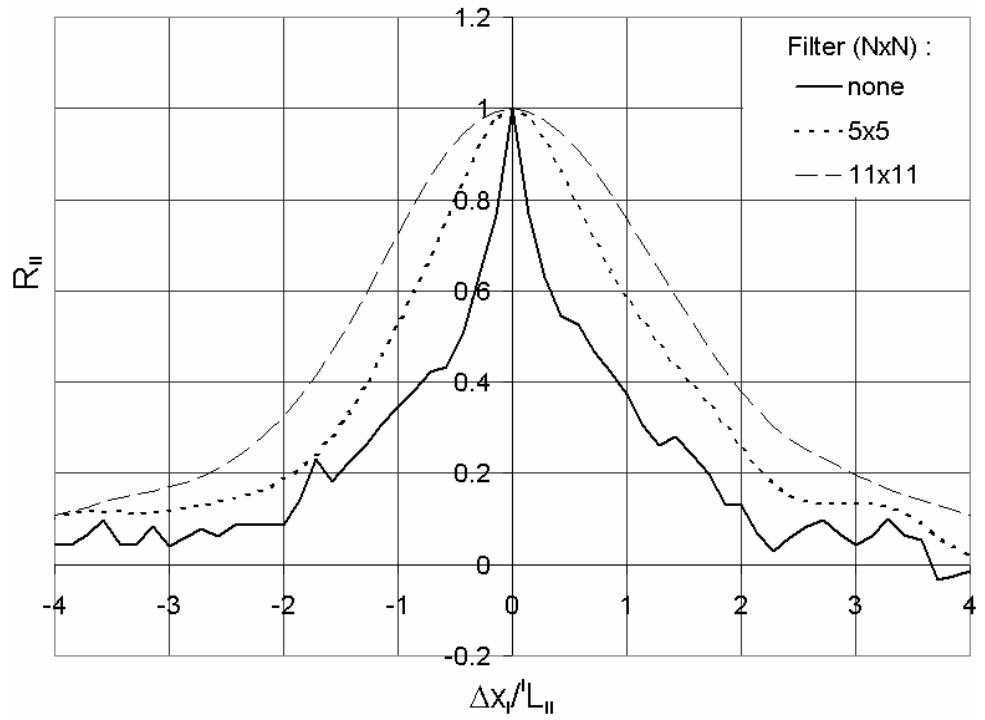

Figure 9. Effect of filter size upon spatial correlation function. 


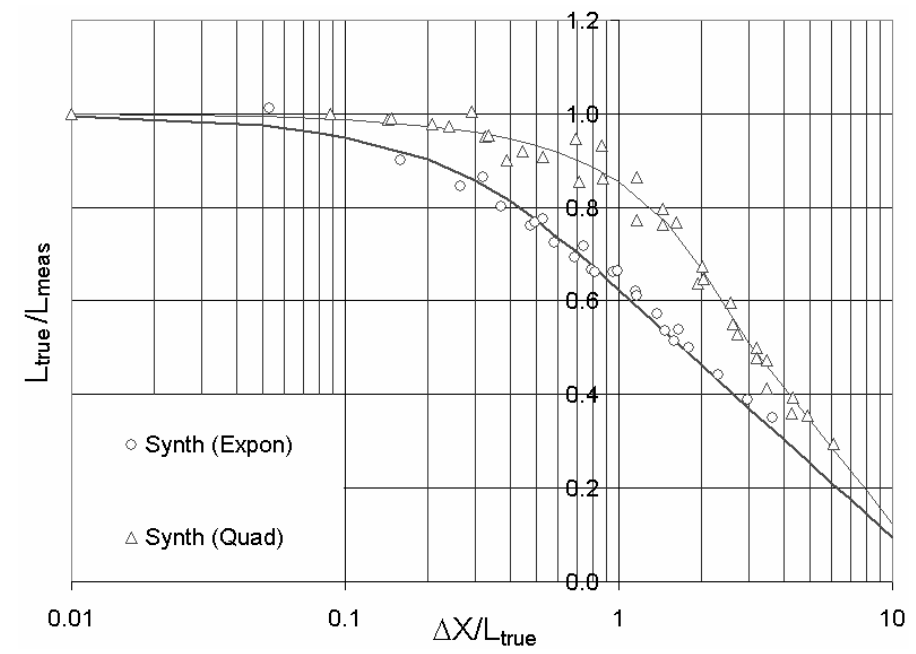

Figure 10. Integral lengthscale trend with increased spatial filtering - synthetic data.

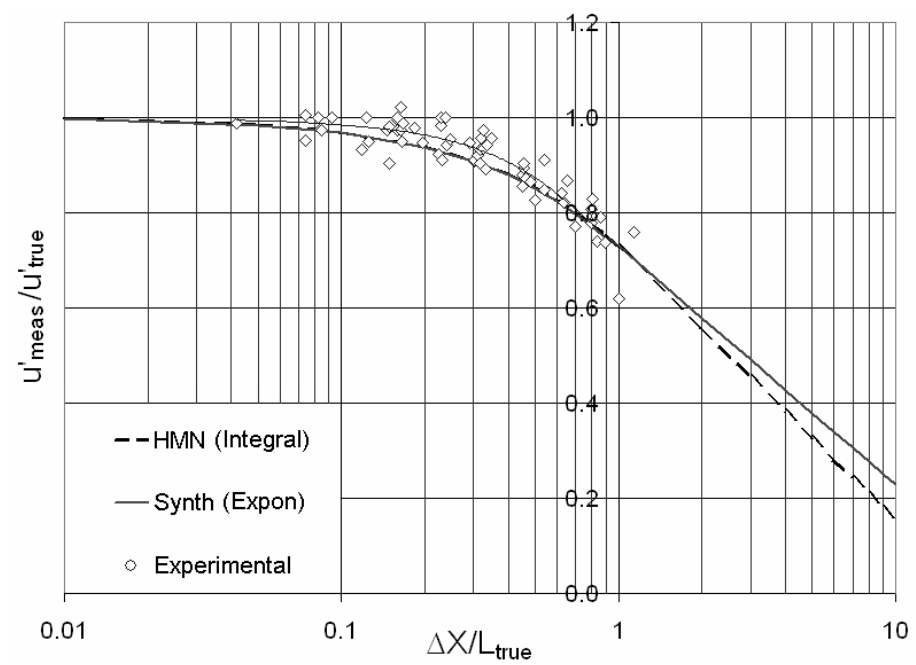

Figure 11. Sub-grid filtering effect on rms velocity - experimental data.

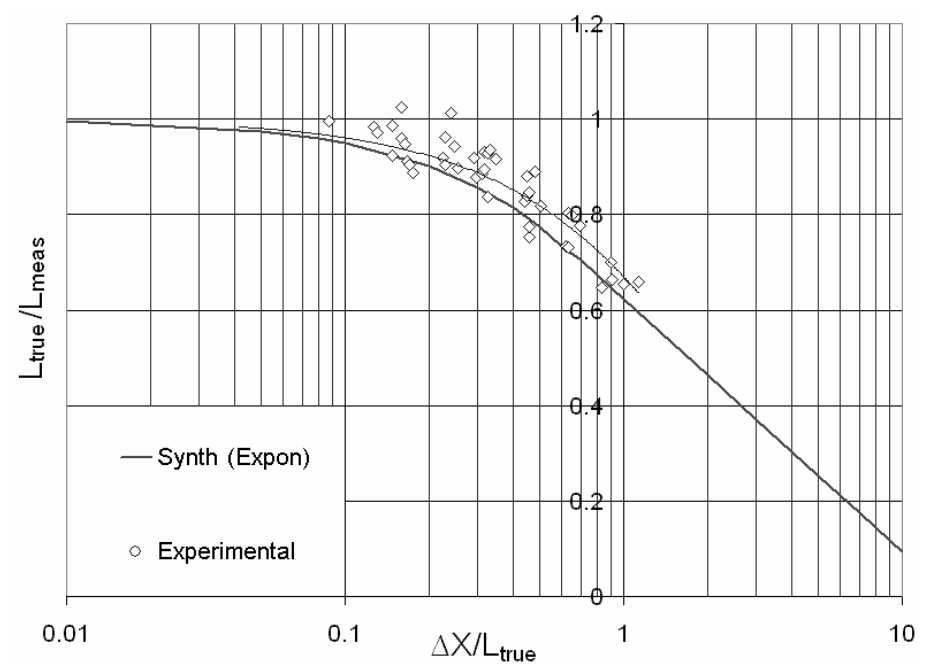

Figure 12. Sub-grid filtering effect on integral lengthscale - experimental data. 


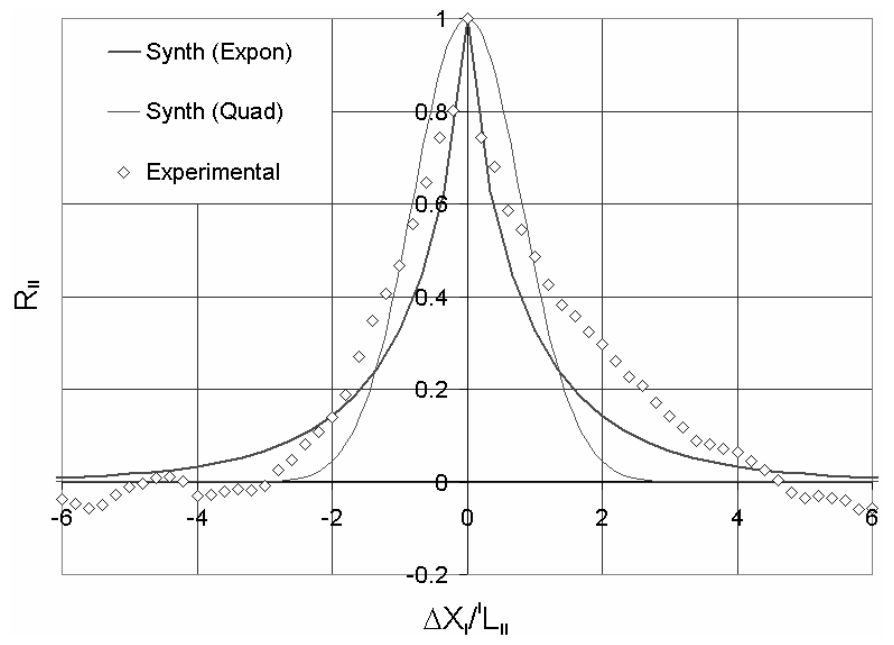

Figure 13. Comparison of experimental spatial correlation function and model functions - Case 2; Point B.

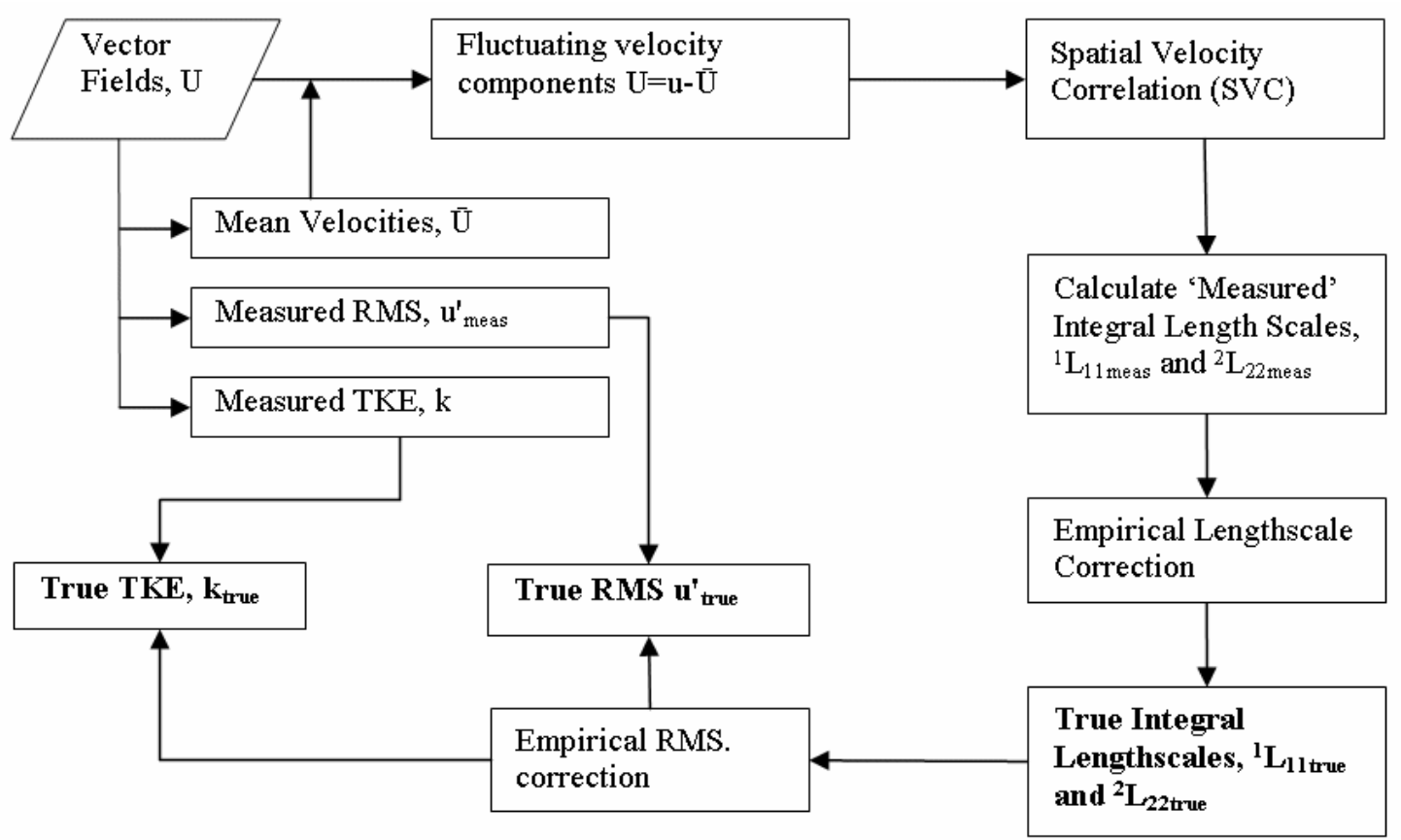

Figure 14. Schematic of sub-grid filtering correction methodology. 


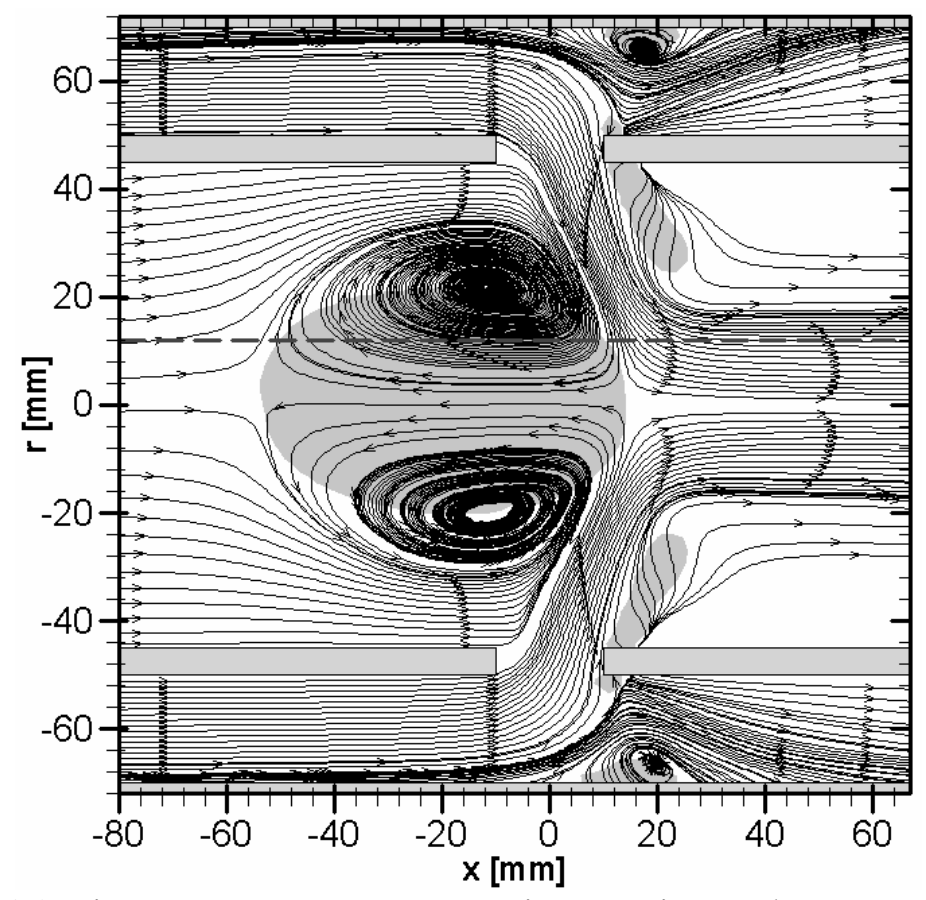

Figure 15. Time average streamtraces in generic combustor geometry. (Shaded contours indicate negative axial velocity.)

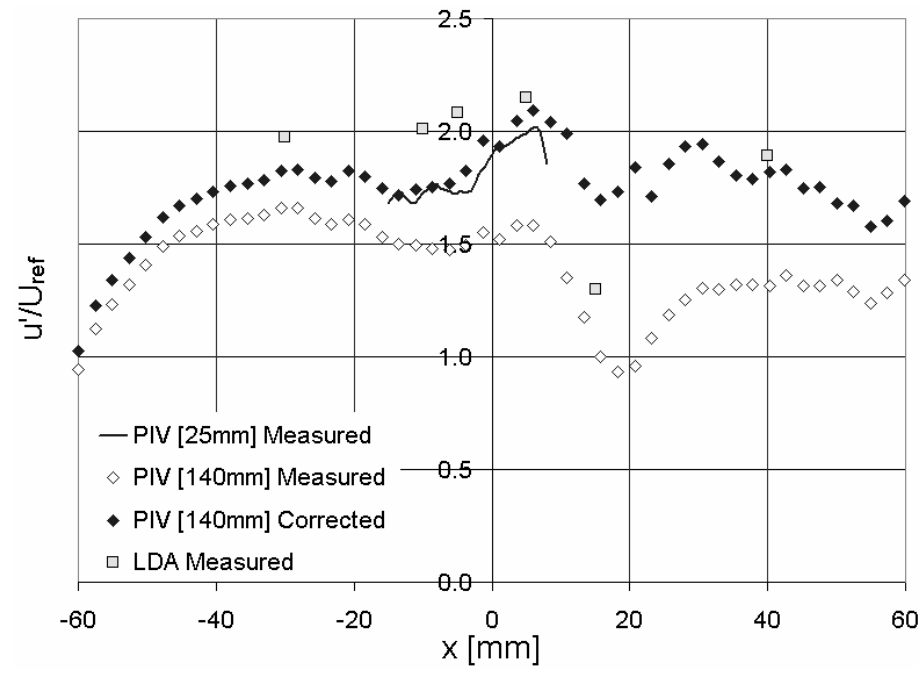

Figure 16. Comparison of corrected and uncorrected data at $\mathrm{r}=12 \mathrm{~mm}$. 


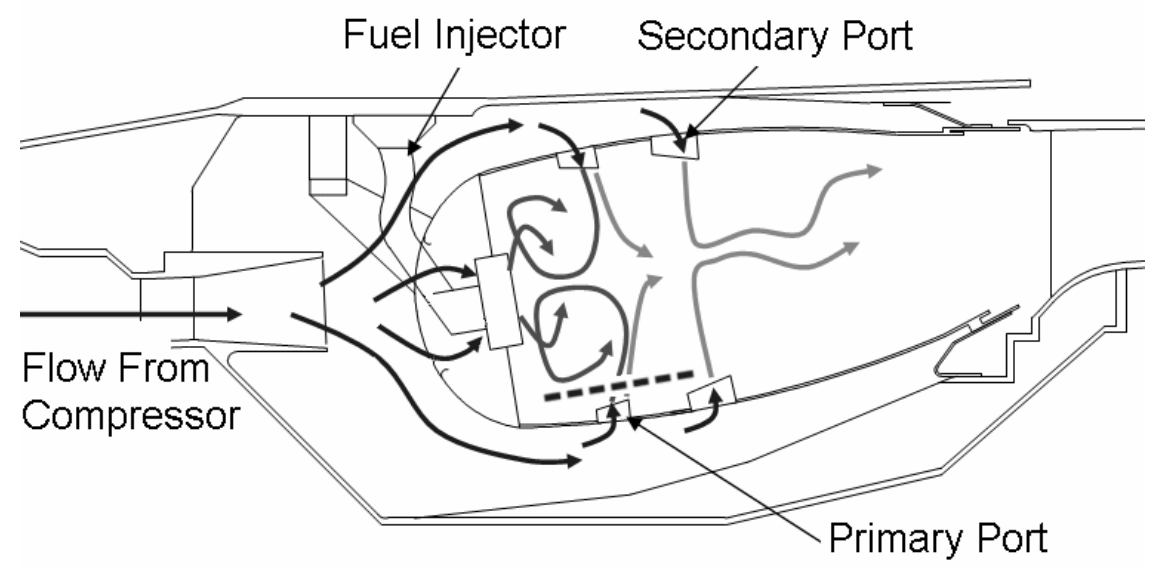

Figure 17. Combustor section view with comparison plot position indicated by the dashed line.

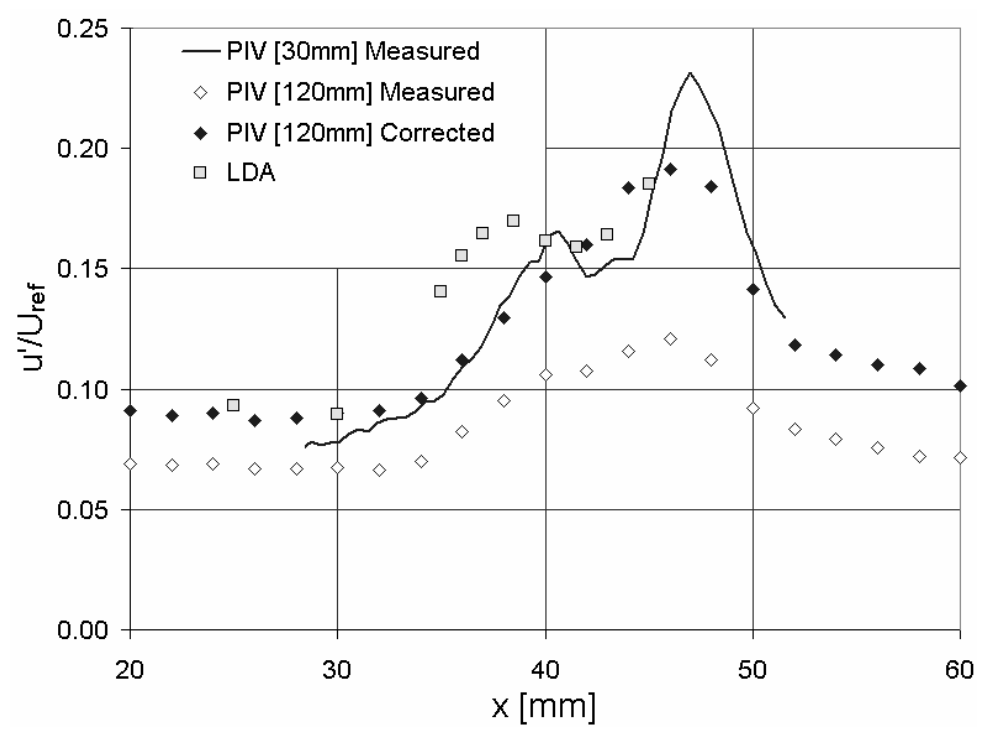

Figure 18. Comparison of corrected and uncorrected data at inner primary port. 